\title{
BLASTOCYSTIS HOMINIS BRUMPT, 1912 (PROTISTA: BLASTOCYSTIDAE) PROTOZOO EN EL TRACTO DIGESTIVO DE LOS SERES HUMANOS
}

Menandro S. Ortiz ${ }^{1}$

\section{SUMARIO}

Blastocystis hominis es un protozoo, organismo unicelular que se halla en el tracto digestivo del ser humano, particularmente en el intestino grueso. Es polimórfica, pero en la literatura existen discrepancias acerca de las formas que presenta, incluyendo la existencia de la forma quística. De igual manera existen discrepancias acerca del poder patógeno que posee. Es anaeróbico y al parecer podría producir malestares, sobre todo cuando existe inmunocompetencia y usualmente en hombres homosexuales y con VIH.

Palabras claves: Blastocystis, protozoo, patógeno, comensal.

\section{SUMMARY}

Blastocystis hominis is a protozoan unicellular organism that is found in the human digestive tract, typically in the large intestine. In literature, discrepancies exist with regards to the various forms (including the occurrence of the cystic form), as well as in the information pertaining to it's pathogenicity. Blastocystis hominis is anaerobic and appears to produce discomfort, especially when immunocompetence is present and usually in HIV infected men.

Keywords: Blastocystis, protozoa, pathogen, commensal organism

1 Facultad de Medicina Humana y Facultad de Ciencias Biológicas. Universidad Ricardo Palma, Av. Benavides 5440, Surco. E-mail: mortiz@urp.edu.pe 


\section{INTRODUCCIÓN}

El medio que nos rodea o donde transitemos, la biodiversidad siempre estará presente representada por poblaciones de especies propias de un lugar determinado o cosmopolitas; y que, pueden ser benéficas o tener un comportamiento de agresión, particularmente a la salud humana. Desde este último punto de vista, es posible encontrar especies que alteren en diferente grado de magnitud la salud del ser humano; ya de manera directa o con seres vivos que se comportan como intermediarios.

En esta oportunidad se trata de la presencia de Blastocystis hominis, una especie de protozoo que habita en el intestino grueso del ser humano, por ende anaeróbico. Se presenta los resultados y/u opiniones de diferentes investigadores acerca del polimorfismo y comportamiento de esta especie, infiriendo que es preciso trabajos más específicos a fin de comprender finalmente el status quo de esta especie. Es posible encontrarlo también en algunos animales (Apt, 2013).

\section{SOBRE EL ROL QUE DESEMPEÑA BLASTOCYSTIS HOMINIS}

Se trata de una especie que pertenece al Reino Protista. Algunos autores lo ubican desde el punto de vista sistemático (de clasificación) en el Reino Chromista, considerándolo como un reino independiente. El grupo en cuestión incluye un buen número de especies de algas; por ello, diferentes autores, entre ellos Salinas \& Vildozola (2007) señalan que existe controversia en cuanto a su posición sistemática, morfológica así como también en cuanto a su patogenicidad.

Zierdt et al. (1967) precisaron su ubicación sistemática como perteneciente a los Protozoa, asignándolo como patógeno primario. Se trata de una especie que se halla con mayor frecuencia en las heces de personas sintomáticas y asintomáticas. De manera similar se expresan Noureldin et al. (1999), como también Uribarren (2014), indicando además que es anaeróbica, polimórfica y de distribución cosmopolita, siendo su prevalencia entre el 30 y $60 \%$ en países en desarrollo. Salinas \& Vildozola (2007) refieren que además de ser anaeróbica, es sensible al oxígeno. Zerpa \& Terashima (2000) lo tratan dentro del ámbito de la amebiosis, sin distinguir el polimorfismo que le atribuyen otros autores.

Esta especie fue descrita por Alexieffer en 1911, tratándola como si fuera un hongo y denominándola en aquella ocasión como Blastocystis entere- 
cola, tal como lo señala Zierdt (1991). Brumpt (1912) tratándola como especie válida, la denominó finalmente como Blastocystis hominis. A ello se agrega el trabajo de Nakamura et al. (1996), quienes descartaron que esta especie sea un hongo. Para mayor información Salinas \& Vildozola (2007) señalan que es un organismo unicelular y anaeróbico que habita el tracto gastrointestinal. Denegri (1999) afirma, tal como lo expresan otros autores que se trata de un protozoo de controvertido poder patógeno que habita en el intestino del hombre y de otros animales, sin señalar la parte del intestino en el que se ubica. Adicionan que son células esféricas de tamaño variable, entre 4 a $15 \mu$, multinucleadas y confirma que son anaeróbicas estrictas. Stenzel \& Borehanm (1996) indican que presentan mitocondrias, aparato de Golgi, retículo endoplasmático liso y rugoso; y que su reproducción es por fisión binaria, desarrollándose en cultivos bajo condiciones anaeróbicas. Apt (2013) resalta que Blastocystis hominis es un protozoo de patología controvertida. Agrega que a la fecha está en duda su papel patógeno.

Su comportamiento en el tracto digestivo, de acuerdo a lo señalado por diferentes autores es controversial. Grosman et al. (1992) le atribuyeron un comportamiento comensal. Posteriormente señalaron su capacidad parasitaria, tal como lo refieren Giacometti et al. (2003) y Barahona et al. (2003). Salinas \& Vildozola (2007) señalan que las infecciones fueron diagnosticadas reconociendo la forma vacuolar de Blastocystis hominis en materia fecal, pudiendo ser ignoradas la presencia de otras formas del organismo. La forma vacuolada es la usualmente más usada para utilizarla para el diagnóstico. Existe gran debate sobre su rol patogénico; por lo que hay trabajos que le otorgan tal actividad, en cambio otros estudios no le asignan tal rol de patogenicidad (Senay \& Macpherson, 1990; Miller \& Minshew, 1988). Sin embargo, Córdova et al. (2009) señalan que Blastocystis hominis, algunas veces se comporta como parásito emergente, oportunista; que puede causar síntomas en gran número de personas.

Uribarren (2014) señala que se ubica en el colon. Además de acuerdo a procedimientos médicos, se ha establecido que no invade la mucosa del colon en seres humanos; dejando establecido que ello depende de la capacidad inmunológica del paciente (Caravelli et al. 1991). Apt (2013) es de la misma opinión, remarcando que no invade la mucosa intestinal.

Devera et al. (2000) manifiestan que Blastocystis hominis puede ser responsable de un cuadro patológico, siempre y cuando se cumplan las si- 
guientes condiciones: que se presente en un gran número en muestras fecales, que existan formas vacuolares grandes, ausencia de otras posibles causas y naturalmente la desaparición de los síntomas posterior al tratamiento específico.

Stenzel \& Boreham (1986) sugieren que la transmisión de Blastocystis hominis se produce a través de la vía fecal - oral, sin descartar el mecanismo de transmisión por la asociación de alimentos contaminados con excretas, tal como lo reiteran Caravelli \& Scaglioni (1989). Uribarren (2014) también hace referencia sobre la forma de transmisión, señalando a la vez que existe gran cantidad de literatura contradictoria sobre la patogenicidad. Finaliza señalando que Blastocystis hominis se le ha relacionado con el síndrome de colon irritado, aunque señala también que faltan estudios clínicos confiables sobre este aspecto.

Teniendo como base lo establecido por Noureldin et al. (1999), al señalar que es una especie polimórfica, Uribarren (2014) agrega que han sido identificado 17 subtipos y que la especificidad del hospedero parece tener alguna relación con el subtipo. Sin embargo agrega que en relación a aspectos morfológicos, los tamaños y formas varían en relación a los hospederos. Concluye que se han caracterizado las siguientes formas: vacuolar, granular, ameboide y quística. De todas estas, continúa, que la forma vacuolar es la más frecuente en heces y la forma quística es la infectante. Así mismo deja entrever sobre la presencia de otras formas como la avacuolar y la multivacuolar. Al margen de lo anteriormente expresado, Salinas \& Vildozola (2007) definen que solo existen nueve subtipos; haciendo ver que la forma granular es similar a la forma vacuolada y que al parecer se trata de ésta última forma con gránulos en la vacuola central. La forma ameboide es usualmente hallada en cultivos viejos o posterior a un tratamiento, según detalla Zierdt (1991). La forma quística presenta resistencia al medio externo, sin embargo no ha sido confirmado experimentalmente (Stenzel y Borehanm, 1996). Por otro lado estos autores indican que la forma presente en el intestino humano parece ser una pequeña célula avacuolar, cuyas pequeñas vesículas presentes en el citoplasma tal vez se adhieren y consecuentemente la célula se presenta como forma multivacuolar. De la misma manera, indican finalmente que la forma quística se halla en materia fecal almacenada en heces frescas; por lo que infieren que esta forma se desarrolla a la salida del hospedero o por factores ambientales externos. Apt (2013) indica que existen las 
siguientes formas: vacuolar, multivacuolar, granular, ameboide y la quística.

Denegri (1999) señala que la reproducción es mediante fisión binaria, endodiogenia y plasmotomía. Adiciona que presenta tres formas diferentes, que son la vacuolar, la granular y la ameboide. Señala además que no se han descrito formas quísticas, concepto contrario a lo que particularmente señala Uribarren (2014). Indica que la forma ameboide es una célula polimorfa con diversos tamaños, presenta pseudopodos y de actividad fagocitica, predominante en cultivos, así como en muestras fecales. Adiciona que la forma granular presenta gran cantidad de mitocondrias, hecho que le otorga tal apariencia, coincidiendo de esta manera lo dicho por Stenzel \& Boreman (1996). Refiere que las formas ameboides y granular derivan de la forma vacuolada. La misma autora señala que no se ha reportado diferencias por sexo sobre esta especie, pero que si existe una mayor tasa de infección en hombres homosexuales. Apt (2013) refiere que posiblemente en pacientes inmunocomprometidos actúa como agente oportunista y puede presentar patogenicidad selectiva. Agrega que en la mayoría de los casos no es patógena, es decir actúa como comensal sin embargo, concluye que la mayor presencia de esta especie ocurre en los homosexuales, así como en pacientes con HIV; pero que aún en tales casos no origina patología.

\section{CONCLUSIONES}

Por todo lo visto, Blastocystis hominis es un protozoo que habita el tracto digestivo, con precisión en el intestino grueso. Los diversos autores que han tratado a esta especie señalan indefectiblemente la presencia del poliformismo, pero algunos de ellos no se ponen de acuerdo cuales son estas; sin embargo se puede señalar que definitivamente predomina la forma vacuolar.

Sobre su presencia en poblaciones relativamente altas, que podría comportarse como patógena, la refieren fundamentalmente para personas inmunodeficientes y hombres homosexuales e incluso con VIH. Aun así no hay consenso sobre el rol patogénico que desempeña Blastocystis hominis. 


\section{LITERATURA CITADA}

Apt W. (2013) Parasitología Humana. Mc Graw Hill Education, México, p. $152-154$.

Barahona RL., Maguiña Vargas C. Náquira C. Terashima IA., Tello R. (2003) Human blastocystis prospective study symptomatology and associated epidemiological factors. Rev. Gastroenterol. Perú, 23: $29-35$.

Brumpt E. (1912) Colite a Tetramitus mesnili (Wenyon, 1910) et colite Trichomonas intestinalis Leuchart, 1879, Blastocystis hominis $\mathrm{n}$. sp. et forms voisines . Bull. Socww. Exot., 5: 725-730.

Cimerman B, Cimerman S. 2002. Parasitología Humana, $2^{\text {da }}$ ed. Sao Paulo. Atheneu, Brasil.

Córdova E, Neira M, Liu M, Vásquez L, Martínez E, Ayaqui R, Ruelas N (2009) Parasitología Humana, $2^{\text {da }}$ ed. Arequipa Perú, p. 75.

Denegri M. in Atias A. (1999) Parasitología Médica. Mediterraneo, Chile, 160- 163.

Devera RA, Velásquez VJ, Vásquez M. Azacón B, Jiménez H. (2000) Blastocystis hominis: criterios de patogenicidad, Saber, Universidad de Oriente, Venezuela, 12: 23-28

Garavelli PL, Scaglioni L. Blastocystosis. An Epidemiological study. Microbiológica, 12: 349-350

Giacometti A, Cirioni O, Antonicelli, D’Amato G, Silvestri C. Del Prete MS, Scalise G. (2003) Prevalence o intestinal parasites among individuals with allergic skin disease. J. Parasitol, 89: 490-492.

Gravelli PL, Scaglioni L. Bicochi R. Libanore M. (1991) Pathogeniicity of Blastocystis hominis. Infection, 19: 185.

Grossman I, Weiss LM, Simon D, Tanowitz HB, Wittner M (1992) Blastocystis hominis in hospital employees. Am. Gastroenterol., 87: 729-732.

Kain KC, Noble MA, Freeman HJ. Barteluk RL (1987) Epidemiology and clinical fetures associated with Blastocystis hominis infection. Diagn. Microbiol. Dis. , 8: 235-244. 
Miller RA, Minshew BH (1988) Blastocystis hominis: an organism in serach of a disease. Rev. Infect. Dis., 10: 930.

Nakamura Y, Hashimotho T, Yoshikawa H, Kamaishi T, Nakamura F, Okamoto K, Hasegawa M (1996) Phylogenetic position of Blastocystis hominis that contains cytochrome free mitochondria, inferred from the protein phylogeny of elongation factor 1 . Mol. Biochem Parasitol, 77: 241-245.

Noureldin MS, Shaltout AA, El Hamshary EM, Ali ME (1999) Opportunistic intestinal protozoal infections in immunocompromised children. J. Egypt Soc. Parasitol. 29: 951-961

Salinas JL. Vildozola H. (2007) Infección por Blastocytosis. Rev. Gastroent. Perú, 27

Senay H, Macpherson D. (1990) Blastocystis hominis: epidemiology and natural history. J. Infect. Dis., 162: 987-990.

Stenzel DJ, Borehanm PF. (1986) Blastocystis hominis Reviseted. Clin. Microbio Rev, 9: 563-584.

Stenzel DJ, Borehanm PF. (1991) A cyst-like stage of Blastocystis hominis. Int. J. Parasitol. 21: 613-615.

Uribarren T. (2014) www.facmed.unam.mx/deptos/microbiologia/ parasitología/blastocitosis.html

Zerpa R y Terashima A. (2000) Blastocystosis. Diagnóstico 2000, 39 (3): $120-122$.

Zierdt CH. (1991) Blastocystis hominis, Pasado y Futuro. Clin. Microbio Rev., 4: 61-79

Zierdt CH, Rude WS, Bull BS (1967) Protozoan characteritics of Blastocystis hominis. An. J Clin. Pathol., 48: 495. 